\title{
La reflexión cotidiana en el proyecto arquitectónico. Hacia un vínculo de la teoría con la práctica y el contexto.
}

Daily reflection in the architectural project. Towards a link theory with practice and context

Laura Gallardo Frías

\section{Filiación}

Universidad de Chile

E mail: lauragallardofrias@uchilefau.cl

Primera versión recibida en: 22 de diciembre, 2015

Última versión recibida en: 29 de diciembre, 2015

\section{Resumen}

En este artículo se describe un proceso proyectual, realizado en el taller de arquitectura de tercer semestre, segundo año, donde se intenta vincular la teoría con la práctica arquitectónica en el contexto específico de cada uno de los proyectos.

Se toma como referencia la vida cotidiana, poniendo al ser humano en el centro del proyecto arquitectónico y, a partir de su ciclo de "reflexión", se propone un camino de investigación hacia diferentes ámbitos de nuestro diario vivir: la calle, el bar y la casa. Instancias que configuran tres ejercicios proyectuales donde va cambiando: la ubicación, los habitantes, el programa, la duración, los conceptos-claves y el grado de dificultad, con la finalidad de realizar propuestas arquitectónicas que cobijen las distintas acciones.

Así, a partir de distintas teorías procedentes principalmente de la arquitectura y la filosofía, tomando en particular como base la obra La "reflexión" cotidiana de Humberto Giannini, se propone mostrar las reflexiones de partida necesarias para poder fundamentar y otorgar un sentido a los proyectos arquitectónicos en su contexto.

\section{Palabras clave}

Proyecto arquitectónico; contexto; reflexión cotidiana; teoría-práctica.

\begin{abstract}
This article describes the process through which second year architecture students attempted to link theory and architectural practice via the development of a studio project.

The main reference for the studio is set on everyday life, which puts the human being in the center of the architectural project. Then, starting from its "reflection cycle", a research path towards different aspects of our daily life is proposed, using street, bar and home as programmatic references to trigger student work. The studio defined three design exercises, which allowed for variation in location, program, expected life time, key concepts and overall complexity, in order to confront the students with an array of different possibilities and constraints throughout the development of their proposals.

Thus, using both philosophical and architectural theories, with Humberto Giannini's Everyday "reflexion" as the main reference, a display of initial ideas as the foundation for architectural meaning is proposed.
\end{abstract}

\section{Keywords}

Architectural project; context; everyday reflection; theory-practice.

\section{Sumario}

Introducción 


\section{REVISTA DE \\ URBANISMO}

ISSN 0717-5051

http://revistaurbanismo.uchile.cl
Revista de Urbanismo N³3 - Julio - Diciembre de 2015 Departamento de Urbanismo - FAU - Universidad de Chile

1 La calle. Lugar de encuentro.

2 El bar. Lugar de comunicación.

3 La casa. Lugar de cobijo.

4 Sinopsis

Bibliografía

\section{Introducción}

Tras la última conferencia en público que hizo Giannini ${ }^{1}$, decidimos realizar nuestro taller de proyectos arquitectónicos, correspondiente al primer semestre del segundo año de la carrera de Arquitectura, basado en los pensamientos del filósofo y lo denominamos: "Arquitectura de lo cotidiano".

Nuestro centro de investigación en taller es el ser humano: habitante y sentido del proyecto arquitectónico, para poder ofrecerle un lugar donde habitar, tomando como marco de referencia: "La vida cotidiana, vida que desde la insignificante apariencia de su superficie ha de abrir el acceso a una reflexión sobre aspectos esenciales de la existencia humana" (Giannini. 2013:28).

Se propone una investigación-acción, relacionando las ideas, el estudio de lo cotidiano y sus posibilidades en el ámbito arquitectónico. Especial desafío de relacionar la teoría con la práctica y generar propuestas arquitectónicas vinculadas estrechamente con sus habitantes y sus experiencias cotidianas.

El objetivo de este escrito es compartir la base teórica procedente de los ámbitos: arquitectónico, filosófico y antropológico que les presentamos a los estudiantes y los proyectos arquitectónicos, y su relación con el contexto, que realizaron a partir de estas reflexiones.

El hilo conductor de nuestro taller, si bien tuvo distintas referencias teóricas, su base principal fue la "reflexión" de los lugares de la vida cotidiana, tomando como referencia los tres hitos planteados por Giannini en su obra La "reflexión" cotidiana (2013): la calle, el bar y la casa, para, a partir de este movimiento circular, revalorizar los momentos de la experiencia común. Tres instancias que configuran el índice de este artículo.

La metodología de diseño proyectual que utilizamos está basada en el planteamiento de tres ejercicios correspondientes a los puntos mencionados: la calle, el bar y la casa, con distintas duraciones: 2.5, 4.5 y 11 semanas respectivamente, realizados en lugares diferentes: barrio Lastarria y parque Forestal en Santiago de Chile, paseo Atkinson de Valparaíso y en el barrio Concha y Toro de Santiago de Chile. Cada uno de estos proyectos tenía un enfoque hacia públicos diferentes en una progresión de lo público hacia lo privado. También con la variación en el encargo, pues el primero era de búsqueda de posibilidades o nichos de acción, el segundo era mixto y en el tercero nosotros les propusimos un encargo a los estudiantes, aunque con un programa flexible. Las etapas metodológicas utilizadas en los tres ejercicios consistieron en comenzar por una revisión teórica de los conceptos relevantes de cada proyecto, seguido de un análisis en profundidad del contexto, para llegar a definir ideas proyectuales que guiaran a cada estudiante en el proceso sus propuestas para llegar a concretizar cada proyecto. Etapas que fueron haciéndose con mayor profundidad en cada ejercicio.

${ }^{1}$ Conferencia realizada por Humberto Giannini para el cierre del Primer Encuentro Nacional de Teoría e Historia de Arquitectura, que tuvo lugar el 14 de noviembre del 2014 en la Universidad Central, Santiago de Chile. 


\section{La calle. Lugar de encuentro.}

\subsection{Reflexiones teóricas en conjunto con los estudiantes.}

Iniciamos el taller con la investigación sobre la vida cotidiana como apertura para el acceso hacia una reflexión sobre los aspectos esenciales de la existencia humana, y los lugares vinculados a ella.

¿Por qué estudiar lo cotidiano? ¿Qué implica la "reflexión" cotidiana? ¿Qué es la "reflexión"? ¿Qué hacemos cuándo reflexionamos?

"Interrogar lo habitual. Pero justamente, estamos habituados a eso. No lo interrogamos, no nos interroga, no parece constituir un problema, lo vivimos sin pensar en ello, como si no fuera portador de ninguna información. Ni siquiera es condicionamiento, es anestesia. Dormimos nuestra vida con un sueño sin sueños. ¿Pero dónde está nuestra vida? ¿Dónde está nuestro cuerpo? ¿Dónde está nuestro espacio?" (Perec. 1992:78)

La cotidianidad es una categoría, un modo de ser, de un ser que, viviendo, se reitera silenciosamente y día a día ahonda en sí mismo. Cotidiano, indica Giannini (2013): es lo que pasa $^{2}$ todos los días, lo que pasa cuando no pasa nada, teniendo en cuenta que el pasar arrastra el sentimiento de que algo queda por pasar.

Todos tenemos una vida cotidiana:...nos levantamos, desayunamos, salimos a la calle... un tiempo reflexivo dado por el "reiterado salir al mundo que tendrá como contrapartida asegurarse un regreso a sí" (Giannini. 2013: 62).

La reflexión implica una "salida y una vuelta" (arriesgando siempre un peligro). La reflexión encuentra siempre algo nuevo. "La reflexión se concluye en un viaje de recogimiento posterior, que consiste, esencialmente, en volver a comprenderse a sí en esta novedad de ser que ha marcado la salida al mundo" (Giannini. 2014:402).

El Ser humano inventa la calle por la reflexión. La vida se reanuda a través de la calle: Lugar del encuentro del desencuentro, de la novedad. Implica un misterio: Comunicación!

La arquitectura también es comunicación, pues vincula barrios, familias....puede hacer una ciudad vivible.

Y en particular, "La calle cumple el oficio cotidiano de comunicar: el lugar del ser para sí (domicilio) con el lugar del ser para los otros (trabajo). Es el medio primario, elemental de la comunicación ciudadana" (Giannini. 2013:41).

Partimos por la calle que aparece como un territorio abierto, límite de lo cotidiano donde suceden encuentros eventuales con la humanidad desconocida. La calle no es sólo medio, también es límite entre distintos ámbitos, de la viabilidad de los proyectos. Se abre un interesante tema de reflexión: el afuera, el espacio público donde nos relacionamos, donde nos encontramos. ¿Dónde se generan los lugares de encuentro en la calle?

Tomamos el barrio Lastarria y el parque Forestal en Santiago para desarrollar este primer proyecto. Tras nuestras reflexiones conjuntas en clase, partimos a las calles que conforman este barrio, empezamos a observar cómo funcionan, las proporciones del ancho de la calle con el alto de los edificios, la relación con los habitantes y usuarios, los árboles, colores, texturas, etc.

\footnotetext{
2 Humberto Giannini recuerda que el término "pasar" es ambiguo en cuanto a su referencia:" porque, por una parte, pasa lo que repentinamente se instala en medio de la vida, lo que irrumpe en ella con novedad ("icqué ha pasado?!). Por otra parte, significa lo fluyente, lo que en su transitoriedad no deja huellas, al menos visibles" (Giannini. 2013:32)
} 


\section{REVISTA DE \\ URBANISMO}

ISSN 0717-5051

http://revistaurbanismo.uchile.cl
Revista de Urbanismo No33 - Julio - Diciembre de 2015

Departamento de Urbanismo - FAU - Universidad de Chile

Realizamos principalmente croquis, fotografías y fuimos haciendo un inventario de lo que había en estas calles, para poder analizar los encuentros.

Nos fueron surgiendo muchas preguntas: ¿Cómo funciona la calle, los distintos ámbitos? ¿Qué nuevas relaciones se podrían establecer? ¿Qué propuestas arquitectónicas se pueden realizar en el barrio para el encuentro de los seres humanos?

\subsection{Programa para el proyecto.}

"Primero, hacer el inventario de lo que vemos. Enumerar aquello de lo que estamos seguros. Establecer distinciones elementales: por ejemplo entre lo que es la ciudad y lo que no es la ciudad". (Perec. 1999:97)

Subrayamos la importancia de las preguntas para la arquitectura, las cuales surgen cuando comenzamos a realizar el inventario:

- Será fundamental la observación para determinar: ¿qué?

- Plantear preguntas, practicar la extrañeza: ¿por qué?

- Realizaremos croquis revisando con mayor atención: ¿cómo?

- Para ir afinando nuestro habitante: ¿para quién?

- Y la ubicación del proyecto: ¿dónde?

Antes de comenzar nuestras propuestas, realizamos un análisis del barrio: Lastarria y Parque Forestal y después, cada estudiante seleccionó un sector donde desarrollar su propuesta.

No dimos un programa específico, sino que cada estudiante, tras el análisis, realizó su propio programa y propuesta proyectual.

\subsection{Propuestas arquitectónicas.}

En este primer ejercicio, de muy corta duración, las dos propuestas seleccionadas se ubicaron en el parque forestal, hay que decir, que la mayoría de los estudiantes trabajó en el parque, quizás por su condición de apertura que invita a mayores posibilidades de acción.

El primer proyecto propuso un paseo a través de una selección de árboles del parque forestal (Fig. 1,2 y 3). El estudiante desarrolló un paseo en altura, para "elevarse" de la ciudad y del parque, y poder contemplar la vida cotidiana desde el plano de las copas de los árboles y revisar sus movimientos, luces, sombras.

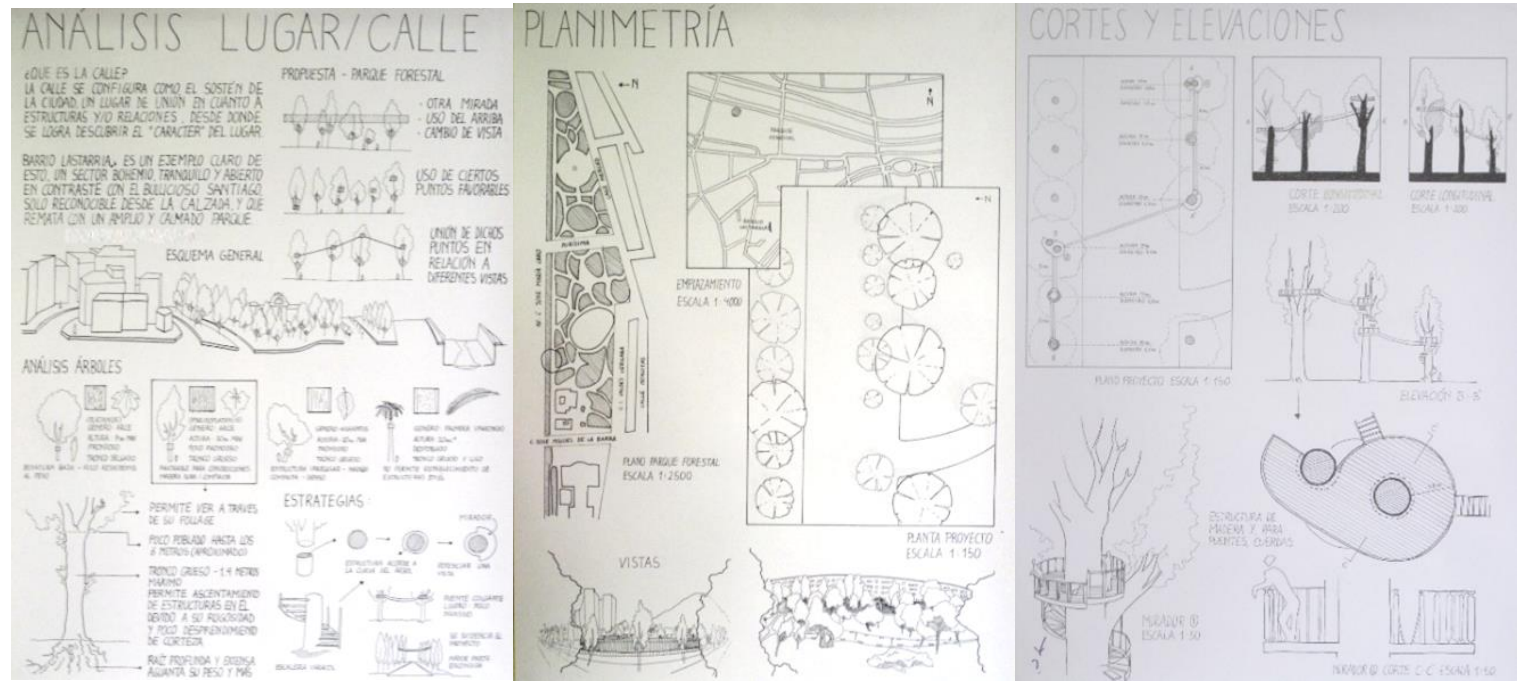

Fig. 1, 2 y 3. Proyecto de paseo entre los árboles.

Fuente: Estudiante Roberto de la Torre. 


\section{REVISTA DE \\ URBANISMO}

ISSN 0717-5051

http://revistaurbanismo.uchile.cl
Revista de Urbanismo No33 - Julio - Diciembre de 2015

Departamento de Urbanismo - FAU - Universidad de Chile

La segunda propuesta consistió en generar una secuencia de gradas siguiendo la topografía del parque forestal, para conformar un teatro al aire libre, un lugar de encuentro para conversar, comer, compartir, abierto a la acción, al movimiento desde el reposo (Fig. 4, 5 y 6)
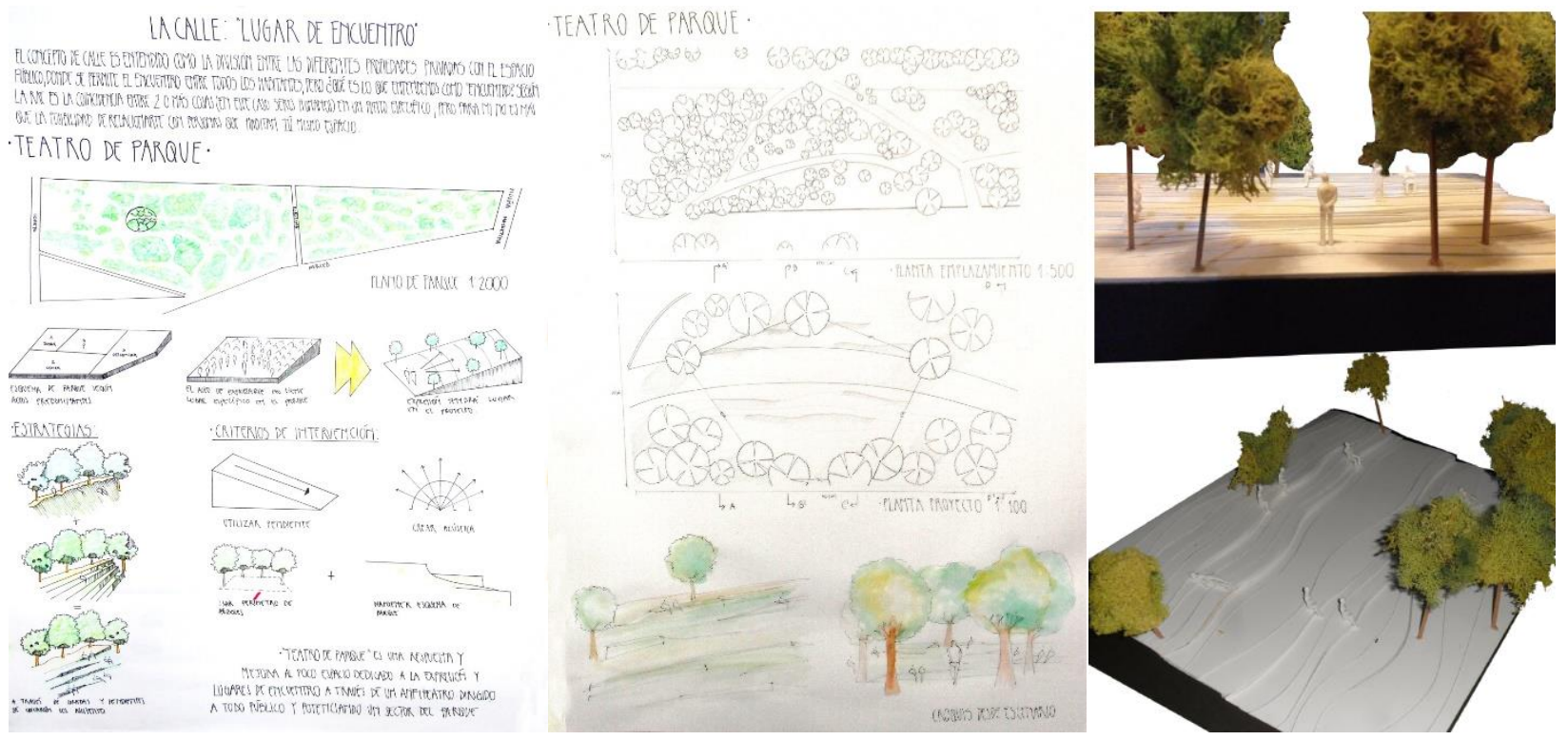

Fig. 1, 2 y 3. Proyecto de teatro parque.

Fuente: Estudiante María Fernanda González.

\section{El bar. Lugar de comunicación.}

\subsection{Reflexiones teóricas en conjunto con los estudiantes.}

Iniciamos la segunda unidad con la revisión de un concepto-clave, base para todos los ejercicios y en general para el ámbito de la proyectación arquitectónica, nos referimos al lugar. ¿Qué es el lugar?

La primera acción fue preguntar a cada estudiante qué entendía por "lugar" y cada uno de ellos lo fue escribiendo en la pizarra. De tal modo que obtuvimos un corpus de definiciones las cuales descubrimos que tenían a su vez muchos términos en común: ser humano, habitar, identidad, encuentro, sentido, cobijo, entre las más destacadas.

Tras esta primera aproximación, donde salieron interesantes reflexiones, estudiamos cuatro definiciones de este concepto a partir de: la filosofía, la arquitectura y la poesía. Las cuales revisamos en una conversación abierta con los estudiantes y que sintetizamos a continuación.

Hegel indica que el espacio y el tiempo no existen por separado, sino siempre en estrecha coordinación. Para el filósofo, "Lugar es tiempo en espacio", "en" equivale a "emplazado", depositado, situado, delimitado, etc. retornando siempre a la idea de que "lugar es tiempo "lugarizado" en espacio" (En Muntañola. 1974:23-24).

Las características que definen un Lugar para Norberg-Schulz, sintetizándolas brevemente, serían: proximidad y cierre, limitado, forma centralizada, relativamente invariante, relación interior-exterior, "el lugar es experimentado como un "interior", en contraste con el "exterior" que lo rodea" (1980:23), haciendo especial hincapié en la identidad, donde parafraseando a Louis Kahn, indica que "identidad significa que los objetos son lo que ellos desean ser". Está íntimamente conectada con la experiencia del lugar, especialmente en los años que se forma la personalidad. El autor expone que lugar es "algo más" que una localización abstracta, es "un 
concreto "aquí" con su identidad ${ }^{3}$ particular" (1981:7). Poniendo de manifiesto el carácter o interacción recíproca con lo que lo rodea: "un lugar es un espacio con un carácter que le distingue" (1981:5). Identidad y carácter, que están íntimamente relacionados con el genius loci o el espíritu del lugar (Norberg-Schulz. 1981).

Juan Navarro Baldeweg afirma que: "La arquitectura se comprende como "una parte de la naturaleza, como un paisaje abstracto, deducido de ella y, además, se dirige a establecer una alianza con el cuerpo entero, con un poder indisoluble. Sus efectos son respaldados por una memoria de innumerables experiencias" (2001:12). Define la Arquitectura a modo de una caja de resonancia que filtra o amplifica los hilos del "tejido continuo y abarcador".

"Lugar" a través del poema Alianza de Alejandra Pizarnik, donde destacamos la "alianza entre lo contemplado y su contemplación (...) La realidad total perceptible en un instante que es todos los instantes (...) $\mathrm{Y}$ el hechizo por un espacio definido: un lugar que obra como llamamiento" (Pizarnik. 2000:418).

Desafío para la arquitectura: hacer emerger un orden de coexistencia del espacio y el tiempo.

Lugar como tiempo asentado en espacio adquiriendo una permanencia que consigue otorgar reposo a lo pasajero, haciendo posible habitar el presente y dotándolo de unas características que lo convierten en único y especial cuya resonancia traspasa los límites físicos impregnando nuestro cuerpo y alma, nuestra percepción y nuestra memoria.

La arquitectura aspira a configurar un receptáculo posibilitando el recibir, abriendo un lugar al ser humano, conexión de interior y exterior, en un instante que son todos los instantes, donde es factible el acontecer.

Así, en esta propuesta el centro fue llegar a generar un lugar que hiciera posible la comunicación, propusimos la realización de un bar o café.

\subsection{Programa para el proyecto.}

Si bien en el ejercicio anterior no había un encargo definido y cada estudiante propuso distintos programas, esta vez les pedimos la realización de un bar o café.

El bar es un lugar de comunicación, desde el exterior suele producirse una invitación a entrar y, una vez dentro, el murmullo de voces nos introduce en un cobijo donde se abre la posibilidad de conversar, compartir, siendo fundamental el encuentro con el otro, los otros para encontrarnos también con nosotros mismos.

Trabajamos en Valparaíso, en el paseo Atkinson, consideramos uno de los terrenos del mirador para la realización de nuestro proyecto y viajamos hasta allá para revisar in situ el sector, con la intención de escuchar el lugar, para formar parte-del contexto. Visitamos un gran número de bares y cafés emblemáticos, revisando sus tipologías, sus diferentes usos de espacios, proporciones, mesas, barras, etc.

Partimos con un análisis grupal del sector, para llegar a definir un concepto o idea sobre la cual generar la propuesta del bar o café. El desafío fue que, siendo un bar, cada propuesta arquitectónica nos ofreciera un cobijo muy especial, donde compartir momentos, un "lugar de comunicación".

El programa específico lo definió en detalle cada estudiante, investigando sobre las necesidades y requerimientos para llegar a conformar un bar, en una estrecha relación con su idea del proyecto.

\footnotetext{
${ }^{3}$ Identidad como característica fundamental que en conjunto con su relación al contexto y la historia, diferencian a un Lugar de un No-Lugar, según indica Augé (2004:83).
} 


\section{URBANISMO}

ISSN 0717-5051

http://revistaurbanismo.uchile.cl

Revista de Urbanismo N³3 - Julio - Diciembre de 2015

Departamento de Urbanismo - FAU - Universidad de Chile

\subsection{Propuestas arquitectónicas.}

La primera propuesta seleccionada, muestra un pliegue en la fachada continua que invitaba a pasar al café-concert. La estudiante cuidó mucho cada ámbito al interior del proyecto: el acceso que generaba una antesala, el escenario, un sector de mesas y una barra en altura, que dialogan entre sí, jugando con la relación entre niveles, configurando diferentes planos de acción y una zona en el segundo piso de comedor que se abre a los distintos ámbitos (Fig. 7 y 8).

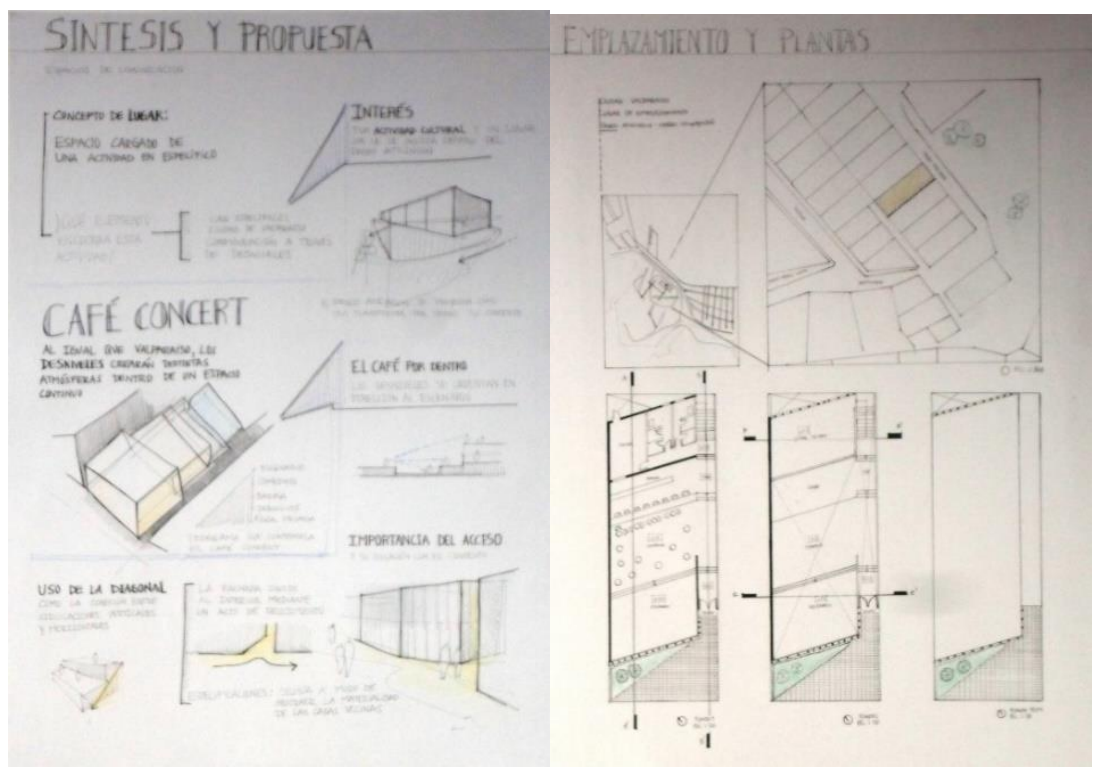

Fig. 7 y 8. Proyecto de café-concert. Fuente: Estudiante Consuelo Jara.
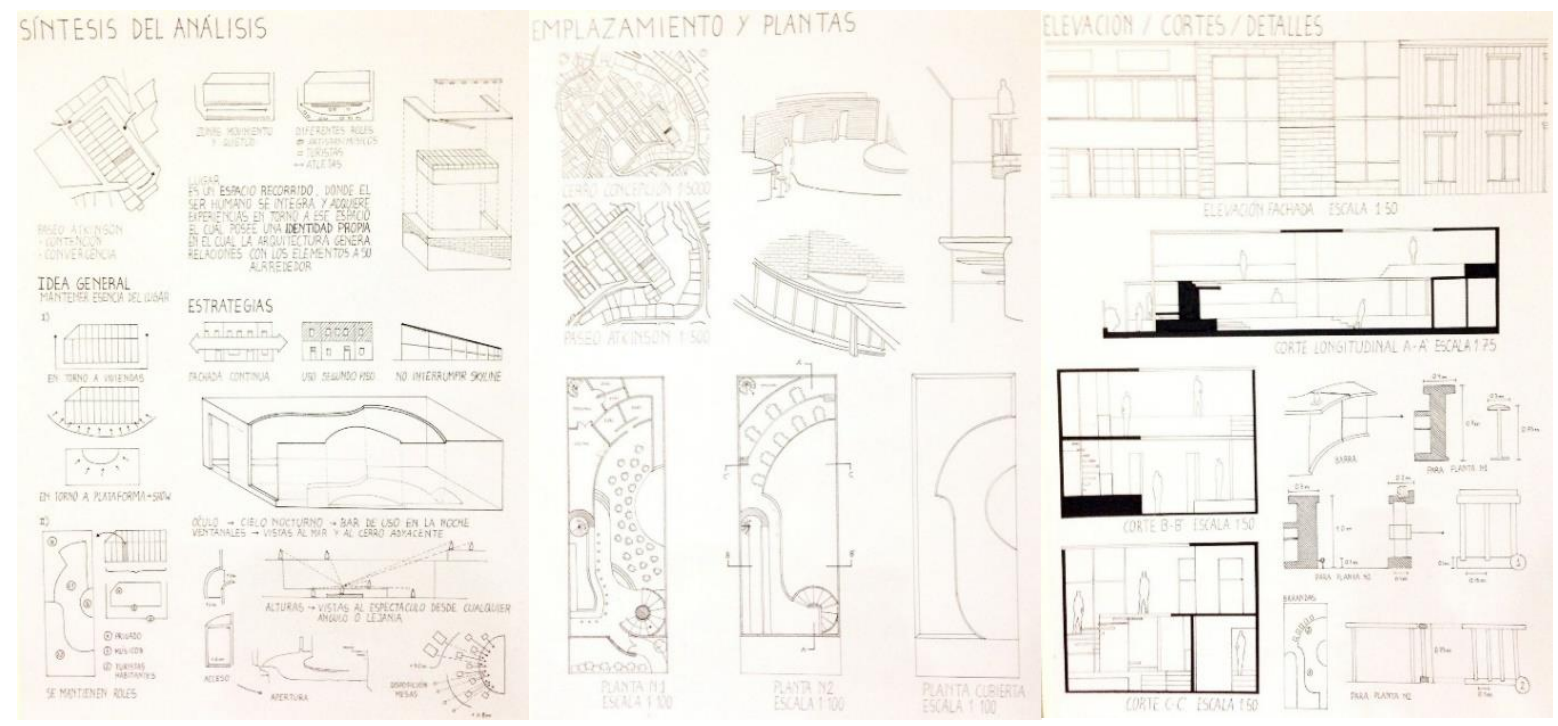

Fig. 9, 10 y 11. Proyecto de bar. Fuente: Estudiante Roberto de la Torre. 
La segunda propuesta mantiene la fachada continua y se organiza en torno a un punto central: el escenario, a partir del cual todo gira, lo que se percibe desde el acceso, configurando el centro de todo el proyecto (Fig. 9, 10 y 11).

\section{La casa. Lugar de cobijo.}

\subsection{Reflexiones teóricas en conjunto con los estudiantes.}

"Cuando traspaso la puerta, el biombo, la cortina que me separa del mundo público; cuando me descalzo y me voy despojando de imposiciones y máscaras abandonándome a la intimidad del amor, del sueño o del ensueño, entonces, cumplo el acto más simple y real de un regreso a sí mismo; o más a fondo todavía: de un regressus ad uterum, es decir, a una separatidad protegida de la dispersión de la calle -el mundo de todos y de nadie- o de la enajenación del trabajo" (Giannini. 2013:35).

El hecho de despertar cada mañana y encontrar la realidad circundante como la dejamos, nos habla de una continuidad espacio-temporal, y se abre la pregunta por el significado del habitar.

Habitar deriva del verbo latino habere (haber) "habere podría llegar a emplearse en lugar de habitare", indica Azara (2005:42) quien prosigue indicando que "ambos verbos tenían la misma raíz, y evocaban una misma visión del mundo. Una habitación es un haber, una posesión, el bien o la posesión más preciada. Habito, en latín, significaba no sólo habitar sino principalmente "tener o poseer a menudo"; por otra parte, el adjetivo habitus se traduce por "saludable", "bien sentado": se trata de un lugar donde se está bien, donde el habitante se centra, se siente seguro, cobijado (Azara. 2005).

Ignasi Solà-Morales en su libro Diferencias. Topografía de la arquitectura contemporánea, define como tarea propia de la arquitectura:"edificar lugares para el habitar", indica que "la arquitectura actúa trazando límites a cielos y tierras que tienen cualidades determinadas.(...) La tarea de la arquitectura es recolectarlas, hacerlas visibles, solidarias, ponerlas en el universo de la palabra. Si el ser humano es el cuidador de las palabras y sólo de ellas emerge el sentido de las cosas, la arquitectura tiene un cometido preciso: hacer de las condiciones ya dadas de cada lugar palabras que signifiquen las cualidades de la existencia, y que desvelen la riqueza y contenidos que en ellas se contienen potencialmente" (Solà-Morales. 1995:115).

En relación al sentido del ser en el proyecto arquitectónico citamos a Heidegger (1997), quien subraya que la existencia es espacial e indica que no puede disociarse al ser humano del espacio. En Construir, Habitar, Pensar, afirma: "Los espacios reciben su esencia de los lugares y no del espacio" (1997:2). A partir de aquí funda el concepto de "residencia", definiéndola como la relación de los seres humanos con los lugares y a través de ellos con los espacios, así, sólo cuando somos capaces de residir podremos construir, ya que la residencia es la "propiedad esencial" de la existencia (Heidegger. 1997).

Pero ¿de qué manera se puede llevar el habitar a la plenitud de su esencia? A lo que responde el filósofo: cuando se construya desde el habitar y se piense para el habitar (Heidegger. 1997).

\subsection{Programa para el proyecto.}

Para el último ejercicio del semestre, y de más larga duración, volvimos a Santiago y tomamos como área de estudio el barrio emblemático de Concha y Toro. El proyecto consistió en la realización de una casa para un habitante que cada estudiante eligió, teniendo en cuenta el análisis en profundidad del contexto.

El desafío fue generar un proyecto arquitectónico donde confluyera el ámbito privado: con una casa, y el ámbito público: con un programa relacionado con el habitante o habitantes y el barrio. 


\section{REVISTA DE \\ URBANISMO}

ISSN 0717-5051

http://revistaurbanismo.uchile.cl
Revista de Urbanismo N033 - Julio - Diciembre de 2015

Departamento de Urbanismo - FAU - Universidad de Chile

Cada estudiante propuso al habitante de su casa, el cual podía ser real o de ficción, con el requisito que lo definiera con exactitud para que todos lo pudiéramos conocer.

Después de nuestras reflexiones sobre la importancia y el significado de la casa y del habitar, pasamos a realizar un análisis en profundidad del terreno en su contexto. Trabajaron en equipos de tres personas revisando 7 puntos de análisis (Gallardo. 2014): genius loci, movimiento-quietud, análisis sensorial, elementos construidos existentes, áreas verdes, y estudio etnográfico. La etnografía fue un punto clave dentro de nuestro análisis, pues nos permitió obtener información de forma directa de las fuentes primarias, esto es, de las personas en su contexto: en el lugar y en el tiempo, a fin de comprender la cotidianidad de ese fragmento, para llegar a realizar proyectos arquitectónicos que se vincularan con sus habitantes y su contexto. El último punto del análisis fue la síntesis, donde se destacaron los principales elementos obtenidos.

\subsection{Propuestas arquitectónicas.}

Les ofrecimos a los estudiantes varios terrenos baldíos del sector Concha y Toro de distintas proporciones.

Entre las propuestas seleccionadas tenemos la casa para un famoso escritor y guionista de comics Stan Lee, el estudiante planteó una casa-estudio para el y su esposa, con un café en planta baja donde se relacionaba el mundo público y privado, abriendo la casa al "mirar" como base de inspiración del habitante. Así, desde todos los ámbitos de la casa, excepto el dormitorio, existen relaciones visuales basadas en distintas formas de mirar, de observar los actos cotidianos del: el café, el cocinar, leer, trabajar, donde ambos mundos público y privado establecen un juego de miradas. (Fig. 12, 13, 14 y 15).

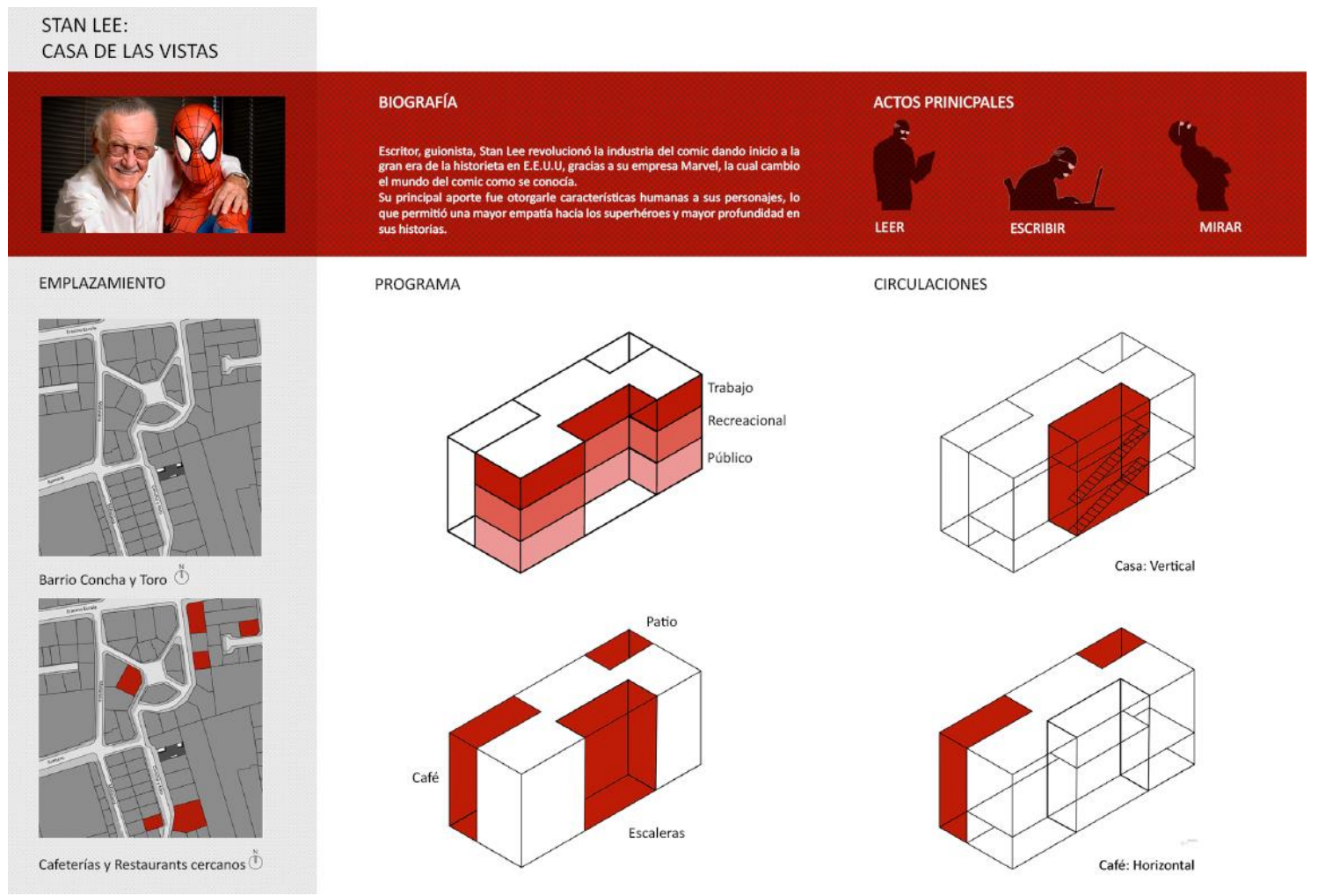

Fig. 12 Proyecto casa de las vistas. Fuente: Estudiante Adrián Cabello. 


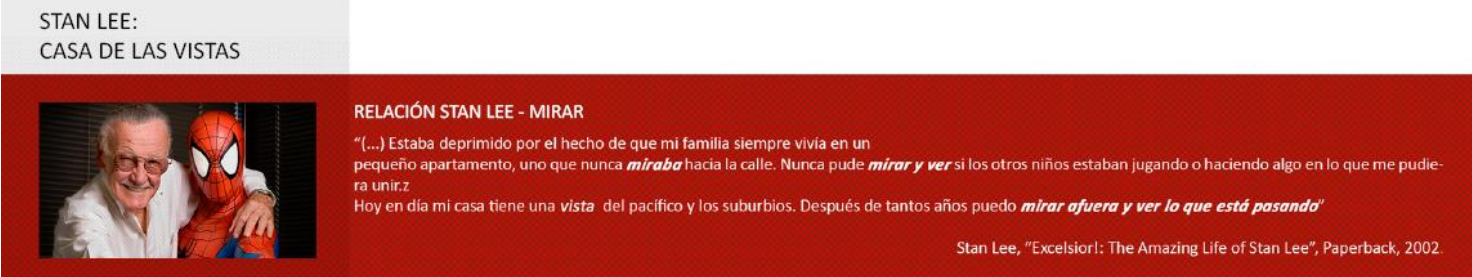

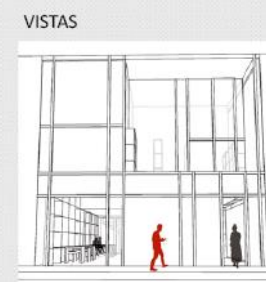

Vista Accesos

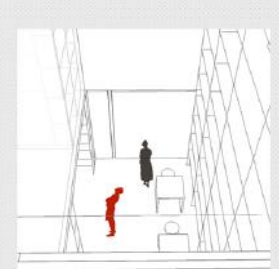

Vista Tercer piso

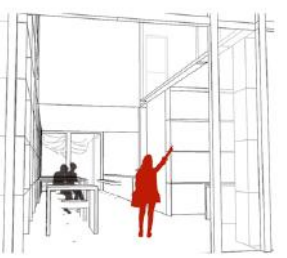

Vista Café

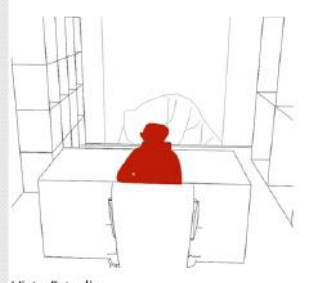

Vista Estudio

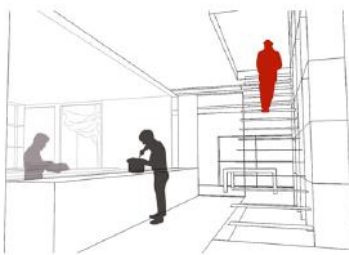

Vista Cocina

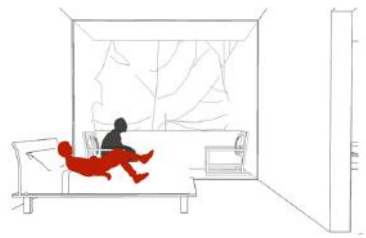

Vista Dormitorio

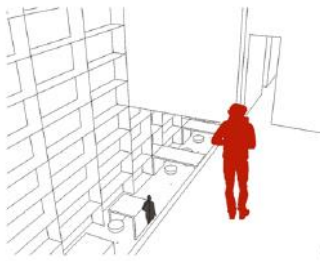

Vista Biblioteca

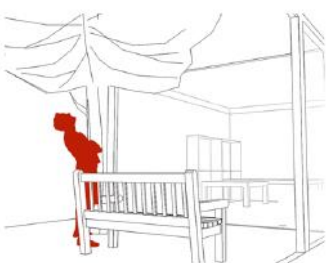

Vista Patio

Fig. 13. Proyecto casa de las vistas Fuente: Estudiante Adrián Cabello.

PLANIMETRIA

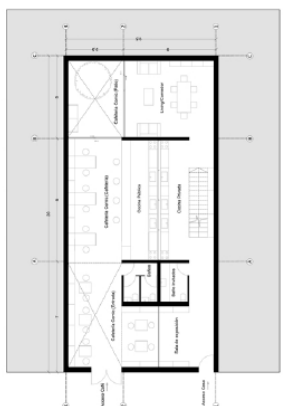

Planta Piso 1 Escala 1:200 ¿

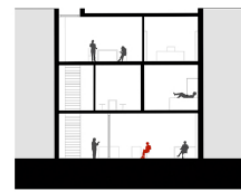

Corte AA' Escala 1:200

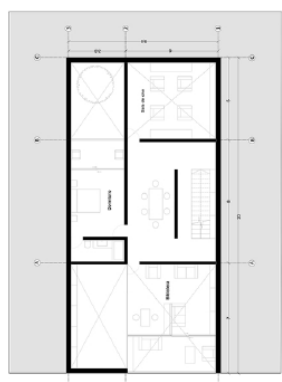

Planta Piso 2 Escala 1:200

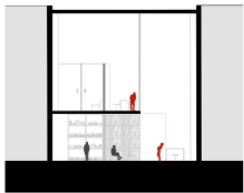

Corte BB' Escala 1:200

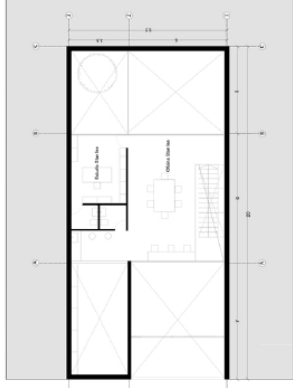

Planta Piso 3 Escala 1:200

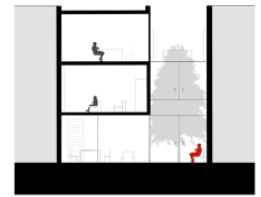

Corte CC' Escala 1:200

Fig. 14. Proyecto casa de las vistas, planimetría Fuente: Estudiante Adrián Cabello. 


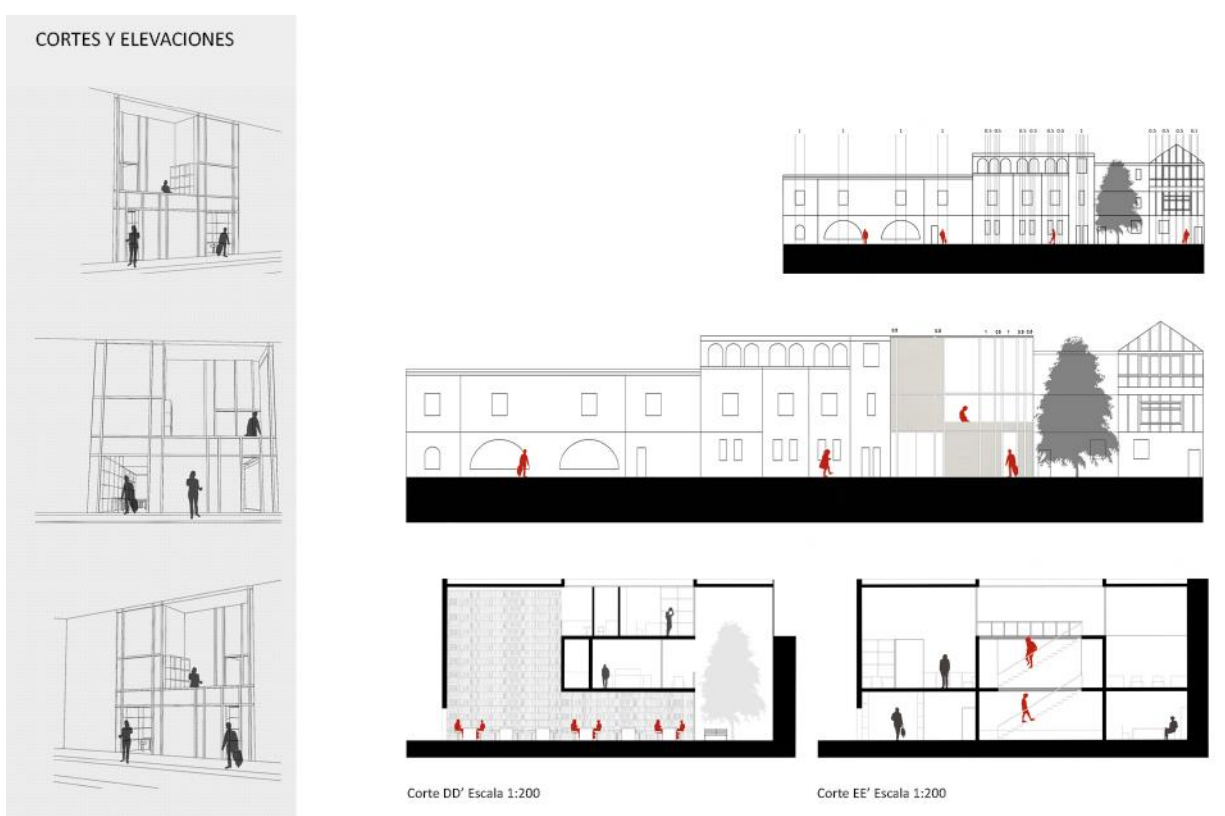

Fig. 15. Proyecto casa de las vistas, planimetría Estudiante Adrián Cabello.

El segundo proyecto seleccionado fue diseñado para Hayao Miyazaki, dibujante de manga y director de cine infantil de animación japonés. La casa partía de una idea muy potente: un árbol, un magnolio japonés, como corazón o centro, donde todo el programa se estructuraba alrededor de él en varios niveles, para ir realizando las acciones cotidianas en un ciclo que se relaciona con las distintas percepciones del árbol, desde la tierra hasta las hojas de sus ramas. La fachada se cierra totalmente en su lado principal, ofreciendo una incisión en el volumen que abre la comunicación al barrio con una pequeña sala de cine (Fig. 16, 17 y 18.)

\section{CASA PARA SOÑAR \\ HAYAO MIYAZAKI}

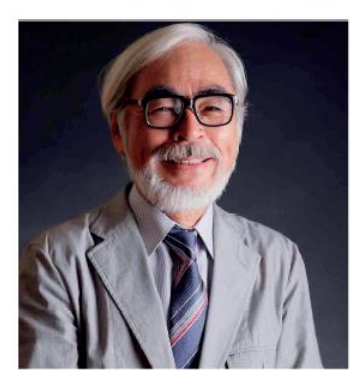

TEMAS RECURRENTES DE SUS PELÍCULAS

-CULTURA JAPONESA

-MUNDO ONÍRICO Y FANTÁSTICO

-NATURALEZA Y PROBLEMAS AMBIENTALES

ACTIVIDADES ASOCIADAS A SUS TEMAS

- SOÑAR

DIBUJAR

OBSERVA

-LEER

DIBUJANTE DE MANGA Y DIRECTOR

DE CINE INFANTIL DE ANIMACION

JAPONÉS

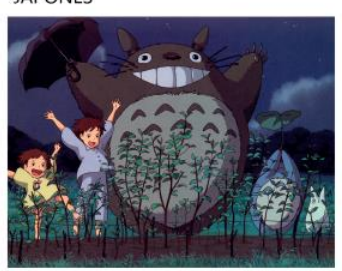

ESTRATEGIAS DE DISEÑO

ÁRBOL CENTRAL QUE ARTICULA Y CONDICIONA EL PROGRAMA DE LA CASA SEGÚN LOS NIVELES DE PERCEPCIOON
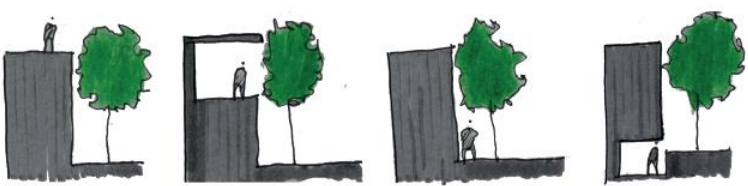

Fig. 16 Proyecto casa Miyazaki.

Fuente: Estudiante Carolaine Muñoz. 
Revista de Urbanismo N³3 - Julio - Diciembre de 2015 Departamento de Urbanismo - FAU - Universidad de Chile

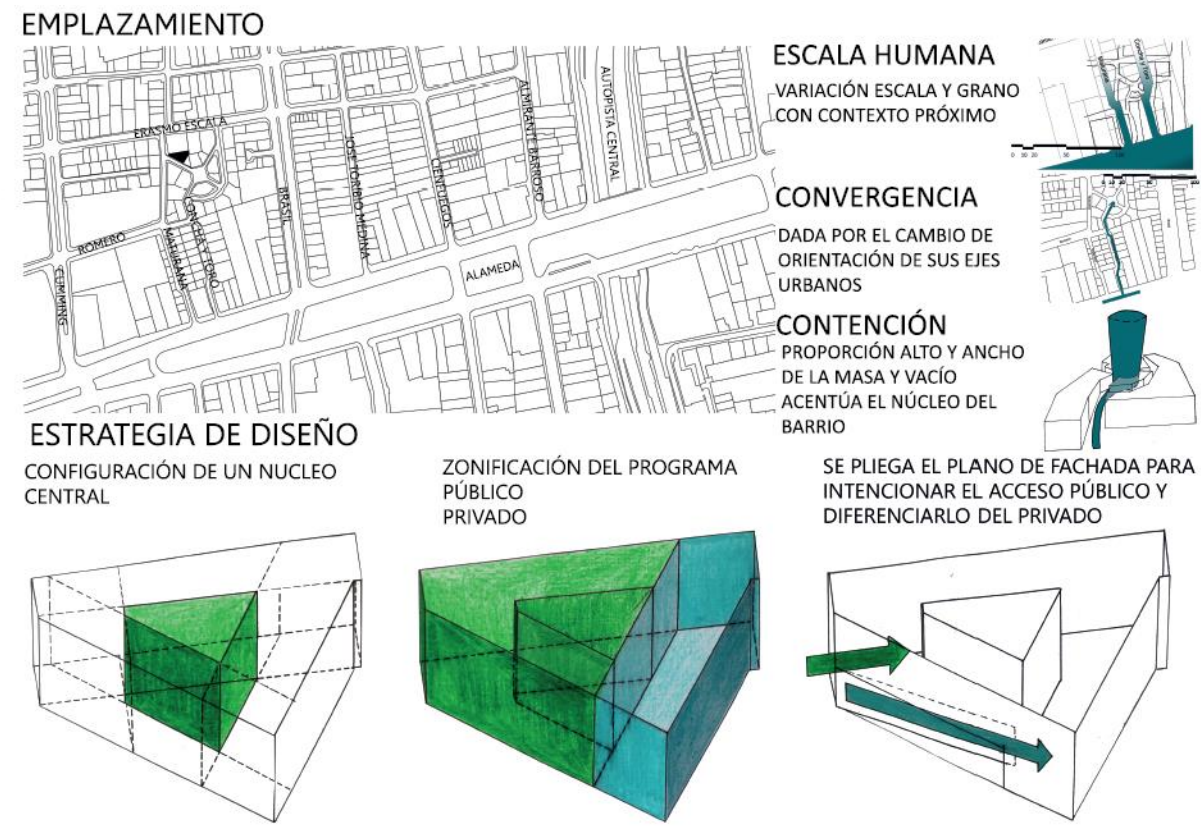

Fig. 17. Proyecto casa Miyazaki.

Fuente: Estudiante Carolaine Muñoz.

PLANIMETRÍA

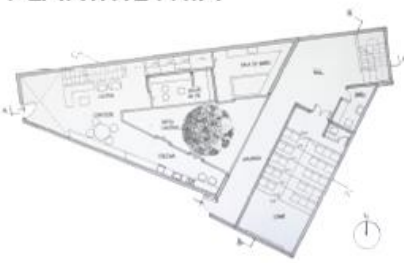

PLANTA NIVEL 1

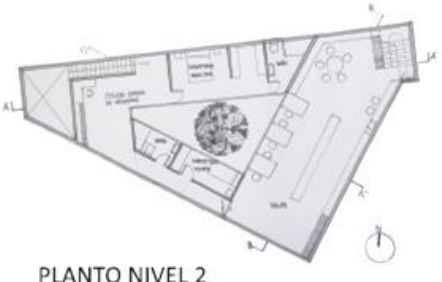

PLANTO NIVEL 2

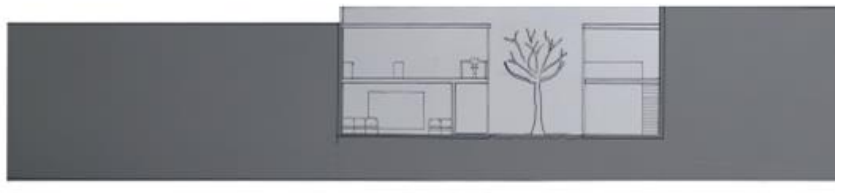

CORTE BB'

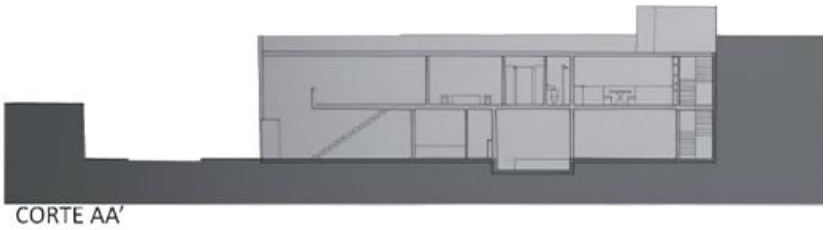

CORTE AA

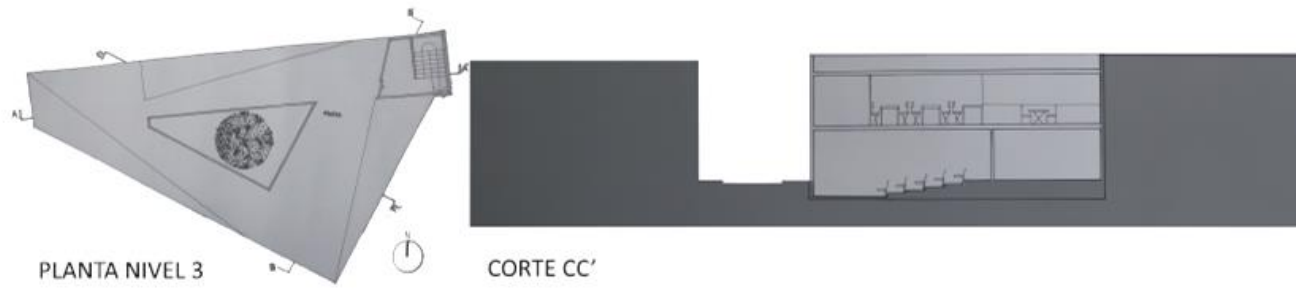

Fig. 18. Proyecto casa Miyazaki.

Fuente: Estudiante Carolaine Muñoz. 


\section{Sinopsis}

Tras la realización de este taller, realizado el primer semestre del 2015 sobre la "arquitectura de lo cotidiano", donde trabajamos con tres proyectos distintos en relación a: sus habitantes, su ubicación, su programa, su duración y sus conceptos-claves, podemos comentar varias cosas con respecto a esta experiencia.

Primero hacer énfasis que nuestros estudiantes, si bien son de segundo año de arquitectura, realizaron grandes reflexiones sobre la importancia del lugar, del habitar y del domicilio al cual se vuelve diariamente.

Fue una sorpresa para todos el adentrarnos en la calle, en el bar y en la casa, lugares de nuestra vida cotidiana, que a primera vista podrían pasar desapercibidos, pero en los que redescubrimos un gran potencial de investigación, de preguntas sobre aspectos esenciales de la existencia humana, que gracias a la filosofía de Humberto Giannini, nos abrieron un horizonte de reflexión en estos aspectos relacionados estrechamente con el proyecto arquitectónico.

Se pone de manifiesto la relevancia del vínculo de la teoría con la práctica, en particular en el ámbito de la proyectación arquitectónica. Lo que consideramos una necesidad, al igual que la interdisciplina, la relación en especial de la filosofía con la arquitectura, también con la poesía y la antropología, entre otros ámbitos.

En nuestro taller hemos podido comprobar que iniciar el proceso de proyectación arquitectónica con reflexiones sobre conceptos relevantes, relacionados con los temas que tratamos, fortalecen enormemente el grado de reflexión de los estudiantes, tomando conciencia de las necesidades del ser humano, del diálogo con su contexto, una pre-disposición teórica que permite fundamentar y otorgar un sentido a los proyectos.

En particular, el maravilloso libro "La "reflexión" cotidiana" (Giannini. 2013), nos guió en la investigación del ciclo cotidiano, a partir de las reflexiones: psíquica, espacial y social ${ }^{4}$ (Giannini. 2012), que nos condujo a estudiar y analizar cada uno de nuestros actos para poder otorgarles un cobijo donde fuera posible el acontecer.

La reflexión hacia estos actos pero teniendo presente el diálogo con lo que existe, con el contexto físico y social que el proyecto arquitectónico debe considerar, teniendo como centro al ser humano. Donde la toma de conciencia del tiempo es esencial: la consideración del pasado desde un presente que se abre hacia el futuro.

La "reflexión" entendida como la incesante búsqueda a partir de lo cotidiano, de los aspectos esenciales que nos proporcionarán ideas inconmensurables (Kahn. 2003) para poder llegar a determinar la esencia del proyecto, que después se pueda construir en la fase mensurable: donde se ordenan el sentimiento y la razón sin descuidar el alma del proyecto en esta etapa, ya que en la siguiente se aspira a volver, una vez que se habita, a aquella idea incomensurable que, desde la quietud nos invite a la inquietud, en esta "reflexión" donde el proyecto arquitectónico adquiere entonces su lugar.

\section{Bibliografía}

AZARA, Pedro, Castillos en el aire. Mito y arquitectura en Occidente, Barcelona, Editorial Gustavo Gili colección Hipótesis, 2005.

\footnotetext{
${ }^{4}$ Giannini habla de tres sentidos del término "reflexión" ligados indisolublemente, por una parte el movimiento reflexivo espacial, relacionado con lo que los psicólogos llaman "ritmo circadiano" correspondiente a las $24 \mathrm{~h}$; después la reflexión psíquica, de una conciencia que se proyecta fuera de sí y luego vuelve sobre sus propios contenidos para interrogarlos y hacerlos transparentes; y por último está la reflexión social que no ocurre esencialmente al espacio sino al tiempo, también relacionada con el "traspaso de mundo" de una generación a otra (Giannini. 2012: 17-18)
} 
Gallardo Frías, Laura, "Siete puntos de análisis en el proceso proyectual. Contexto Urbano en el Proyecto Arquitectónico", en Revista Bitácora urbano/territorial. Colombia. Volumen II. N.25, 2014, p.31-41. ISSN: 0124-7913.

GIANNINI, Humberto, Breve historia de la filosofía, 25a edición, Santiago de Chile, Editorial Catalonia, 2014, 434 págs., ISBN 978-956-8303-23-5

GIANNINI, Humberto. La "reflexión" cotidiana. Hacia una arqueología de la experiencia, $1^{\text {a }}$ edición, Santiago de Chile, Editorial Universidad Diego Portales, 2013, 363 págs., ISBN 978-956-314-226-6

GIANNINI, Humberto. La metafísica eres tu. Una reflexión ética sobre la intersubjetividad. $2^{a}$ edición, Santiago de Chile, Editorial Catalonia, 2012, 170 págs., ISBN 978-956-8303-81-5

HEIDEGGER, Martin. Construir, habitar, pensar. Buenos Aires, Alción Editora, 1997.

KAHN, Louis. I, Forma y diseño. $1^{a}$ ed. 10a reimp, Buenos Aires, Editorial Nueva visión, 2003, 64 págs., ISBN 950-602-073-6.

MUNTAÑOLA T. Joseph, La arquitectura como lugar, Barcelona, Editorial Gustavo Gili, 1974.

NAVARRO BALDEWEG, Juan. Navarro Baldeweg, Sevilla, Tanais Ediciones, 2001.

NORBERG-SCHULZ, Christian. Existencia, Espacio y Arquitectura, Nuevos caminos de la arquitectura, Barcelona, Editorial Blume, 1980.

NORBERG-SCHULZ, Christian, Genius Loci, París, Pierre Mardaga editeur, 1981.

PEREC, Georges, Especies de espacios, Barcelona, Editorial Montesinos, 1999.

PEREC, Georges, Tentativa de agotar un lugar parisino, Rosario, Beatriz Viterbo Editora, 1992.

PIZARNIK, Alejandra, Poesía completa, 8a edición, Buenos Aires, Editorial Lumen, 2000, 470 págs., ISBN 84-264-2825-8.

SOLÀ-MORALES, Ignasi. Diferencias. Topografía de la arquitectura contemporánea. Barcelona, Editorial Gustavo Gili. 1995. 